https://doi.org/10.24201/aap.2021.320

ARTÍCULO

\title{
Derechos sexuales y seguridad en Indonesia ${ }^{1}$
}

\section{Sexual Rights and Security in Indonesia}

SHARYN GRAHAM DAVIES

https://orcid.org/0000-0001-5348-4051

Monash University, Australia

Recepción: 11 de mayo de 2020

Aceptación: 18 de febrero de 2021

Resumen: Los derechos sexuales son derechos humanos fundamentales que los ciudadanos esperan ejercer cuando viven en una democracia que funciona correctamente. Si bien Indonesia ha sido una nación democrática durante más de dos décadas, el acceso a los derechos sexuales es limitado, como podría entenderse en un contexto global. Por ejemplo, existe poca protección legal para los adultos que tienen relaciones sexuales consensuadas fuera del matrimonio heterosexual, e incluso dentro del matrimonio a las mujeres les resulta difícil buscar la justicia sexual, aun cuando ha ocurrido una violación marital. Este artículo explora la relación de Indonesia con los derechos sexuales y la seguridad, centrándose en los años posteriores a 1998, cuando se introdujo la reforma democrática. El artículo utiliza la noción de derechos sexuales para referirse a la capacidad de los adultos consintientes para que sus derechos sexuales sean protegidos, incluida la libertad de la coerción sexual, la capacidad de mantener relaciones sexuales consensuadas, la capacidad de acceder a una educación sexual de calidad y el libre acceso a los servicios de salud reproductiva. El artículo describe los cambios en la relación de Indonesia con la sexualidad, revelando las medidas

\footnotetext{
${ }^{1}$ Muchas gracias a los dos revisores anónimos que dedicaron su tiempo a proporcionar comentarios útiles para enriquecer este artículo. 
cada vez más draconianas utilizadas para controlar la sexualidad (por ejemplo, los agentes de policía pueden entrar legalmente en hogares privados en algunas provincias para verificar los certificados de matrimonio de las parejas). Con un enfoque en la policía, el artículo deja en claro que actualmente en Indonesia las prácticas sexuales no son un asunto privado, sino más bien un asunto de vigilancia estatal abierta y, a menudo, punitiva, por parte de la policía.

Palabras clave: sexualidad; derechos sexuales; seguridad; vigilancia; policía; Indonesia.

Abstract: Sexual rights are one of the fundamental human rights citizens expect when they live in a well-functioning democracy. While Indonesia has been a democratic nation for more than two decades, there is limited access to sexual rights as might be understood in a global context. For instance, there is little legal protection for consenting adults who have sex outside heterosexual marriage, and even within marriage women find it hard to seek sexual justice, even when marital rape has occurred. This article explores Indonesia's relationship with sexual rights and security, focusing on the years after 1998 when democratic reform was introduced. The article uses the notion of sexual rights to refer to the ability of consenting adults to have their sexual rights protected, including freedom from sexual coercion, ability to fulfil consenting sexual relationships, ability to access to quality sexual education, and free access to reproductive healthcare. The article charts changes in Indonesia's relationship to sexuality, revealing the increasingly draconian measures used to literally police sexuality (e.g. police officers are lawfully able to enter private homes in some provinces to check the marriage certificates of couples). With a focus on police, the article makes clear that currently in Indonesia, sexual practices are not a private matter, but rather a matter for overt and often punitive state surveillance, often by police.

Keywords: sexuality; sexual rights; security; surveillance; police; Indonesia.

\section{INTRODUCCIÓN}

Mediante varias leyes propuestas, Indonesia parece estar ahora a punto de criminalizar toda actividad sexual fuera del matrimonio heteronormativo. Sin embargo, los primeros años de 
la democracia en Indonesia sugerían que los derechos sexuales mejorarían, en particular para las mujeres y la comunidad de lesbianas, gays, bisexuales y transexuales (LGBT) (Paddock y Suhartono 2019).

Se realizaron numerosos avances en esta dirección. Por ejemplo, la Comisión Nacional para la Violencia contra la Mujer fue establecida mediante el Decreto Presidencial núm. 181/1998 y reforzada por el Decreto Presidencial núm. 65/2005. La democracia hizo posibles tales avances, pero también dio voz a los conservadores e islamistas que han trabajado durante las últimas dos décadas para impulsar una agenda antirreformista, a menudo bajo el manto de la democracia (Davies y Wijaya y 2019).

Este artículo explora la expresión sexual en Indonesia con un enfoque particular en cómo la vigilancia (incluso por parte de la policía) ha evidenciado una reducción radical de los derechos sexuales, a menudo con consecuencias devastadoras. Para establecer este argumento, el artículo se divide en dos secciones principales. La primera examina el contexto de la represión de los derechos sexuales en Indonesia durante las últimas dos décadas y explora la noción de moralidad populista. La segunda examina la fuerza policial con el fin de entender cómo la policía restringe los derechos sexuales y alimenta la noción de moralidad populista. Con la primera sección se presenta una comprensión del contexto social; la segunda sección la forma en que fuerzas de seguridad como la policía regulan la sexualidad. Lo anterior hace evidente la contribución clave del artículo. Esta contribución es que un clima de pánico moral sobre la sexualidad permite y justifica la acción policial que regula la sexualidad. Para concluir, se analiza el impacto que tiene en los ciudadanos indonesios la falta de derechos sexuales. En resumen, el artículo sostiene que hay poca protección de los derechos sexuales en la Indonesia contemporánea y que es probable que la situación empeore y tenga una repercusión cada vez más devastadora no sólo en las minorías sexuales sino también en todos los indonesios. 


\section{LA MORALIDAD POPULISTA Y LA REPRESIÓN \\ DE LOS DERECHOS SEXUALES}

\section{Esperanza democrática}

En 1999, Indonesia se convirtió en la tercera democracia más grande del mundo. Después de haber derrocado con éxito al líder autoritario, el presidente Suharto, que había gobernado durante tres décadas, Indonesia marcó el comienzo de un sistema de reforma democrática, conocido como reformasi. Éste comprendió una época de gran ansiedad, pero también de esperanza. Suharto había sido derrocado porque su régimen había supervisado una corrupción insoportable, connivencia, nepotismo y flagrantes violaciones a los derechos humanos. Por ejemplo, en el colapso del régimen de Suharto, cientos de mujeres chinoindonesias fueron violadas y torturadas (Andajani-Sutjahjo, Bennett y Davies 2018). En parte para intentar reparar el daño causado, en 1998 se estableció la Comisión Nacional para la Violencia contra la Mujer. Algunos años más tarde, la violación conyugal se convirtió en un delito penal (Bennett, Andajani-Sutjahjo e Idrus 2011). El gobierno reformasi también reforzó los poderes de la Comisión de Derechos Humanos. Además, se introdujeron nuevas leyes para apoyar el sistema de justicia penal (Putri et al. 2019), incluida la justicia de menores, aunque estos sistemas aún están lejos de ser perfectos (Davies y Robson 2016). El periodo inicial de reformas vio una afluencia de fondos de agencias extranjeras, como las Naciones Unidas y la Organización Mundial de la Salud, destinados a ayudar con problemas relacionados con la sexualidad y brindar apoyo en torno a la prevención y el tratamiento del VIH (Davies y Wijaya 2019).

Los primeros años de la reforma democrática brindaron un espacio para una conversación pública sobre los derechos y la diversidad sexuales. Las discusiones sobre género y diversidad sexual y pertenencia nacional también comenzaron en serio (Boellstorff 2005; Hegarty 2018). Sin embargo, debe tenerse en cuenta que la diversidad sexual y de género no eran conceptos extraños en Indonesia ni fueron importados al por mayor desde Occidente. Indonesia tiene su propia historia rica en diversidad sexual y de género (Davies 2018a). No obstante, lo que hizo la reforma democrática fue proporcionar una plataforma 
pública para la discusión de los derechos sexuales de una manera nunca vista en Indonesia y una plataforma pública en este país da acceso a millones de personas.

Indonesia es el cuarto país más poblado del mundo, con alrededor de 264 millones de personas repartidas en 6000 islas habitadas. Se hablan alrededor de 300 idiomas. Esta diversidad geográfica y lingüística significa, sin embargo, que la difusión de información es desigual. Los centros urbanos como Yakarta, Bandung, Yogyakarta y la isla de Bali registraron el mayor aumento en términos de derechos sexuales, como el acceso al tratamiento del VIH. En las islas periféricas, los discursos sobre los derechos sexuales y las habilidades y el dinero para desarrollarlos se sintieron poco. Por ejemplo, los derechos sobre la sexualidad de las mujeres siguieron siendo poco tratados en lugares como Papúa Occidental, que sigue teniendo una de las tasas más altas de violencia sexual contra las mujeres en Indonesia (Toomistu 2019a). Hubo experiencias similares en las islas Mentawai (Towner y Davies 2019). La influencia de la religión, especialmente el islam, a la que se adhiere casi 90\% de la población de Indonesia, también moldeó fuertemente las respuestas a las discusiones sobre los derechos sexuales. A principios de la década de 2000, si bien la democracia había brindado espacio para discusiones progresistas sobre la sexualidad, también, quizá irónicamente, proporcionó espacio para una reacción conservadora contra los derechos sexuales. Gran parte de esta reacción fue impulsada por una agenda islamista. A principios de la década de 2000, el movimiento de moralidad populista había comenzado a encenderse en serio.

\section{Moralidad populista}

La moralidad populista es un término que se usa para pensar colectivamente numerosos movimientos de Indonesia enfocados en la negación de los derechos sexuales (Davies 2019a). El populismo, para recurrir a la definición de Cass Mudde que Marcus Meitzner utiliza en su trabajo sobre Indonesia, es una ideología levemente centrada ${ }^{2}$ que considera que la sociedad se divide, en última instancia, en dos campos homogéneos y antagónicos, el pueblo "puro" y lo Otro, y promociona la idea de que la política debería ser una expresión de la voluntad

\footnotetext{
2 Thin-centered ideology.
} 
general del pueblo "puro" (Mietzner 2020). La gente "pura" en Indonesia, al parecer, a menudo se enmarca como musulmana, casada, monógama y de clase media.

El populismo pasa a primer plano a través de líderes populistas, que no son escasos en el mundo. Respecto a Indonesia se puede decir que el actual presidente, Joko Widodo, es un líder populista (Mietzner 2020). Si bien Joko Widodo no es necesariamente anti-LGBT, el actual vicepresidente, Ma'Ruf Amin, ha hablado con frecuencia en contra de las personas LGBT. Para Ma'Ruf Amin, las personas LGBT son representadas como amenazantes y desestabilizantes de la posición de las personas "puras". Pero no sólo se enmarca como “enemigas" a las personas LGBT, sino a cualquiera que tenga relaciones sexuales fuera del matrimonio y, de hecho, a cualquiera que pueda considerarse que piense en el sexo fuera del matrimonio.

El apoyo al populismo en Indonesia se ha hecho evidente no sólo en el hecho de que Joko Widodo fue elegido para un segundo mandato, sino también en que su oponente en ambas campañas fue un líder populista por excelencia (Mietzner 2020). Sin embargo, podría no ser necesariamente que los votantes estén votando por el líder real. Los votantes podrían estar más bien desilusionados con un mal social más profundo (cf. Mc Williams 2016). De manera similar, parece que la retórica de la represión sexual desatada por los islamistas y nacionalistas en Indonesia no está precisamente en el centro del odio y la violencia resultantes hacia las minorías sexuales y otras. Más bien, la violencia podría ser un síntoma de una enfermedad más profunda. Para Mietzner, esta enfermedad es un endurecimiento de las políticas de identidad entre grupos que sienten que su lugar en la sociedad está siendo amenazado (Mietzner 2020). En Indonesia, para los islamistas, nacionalistas y otros, la amenaza se posiciona cada vez más como proveniente de aquellos que se consideran sexualmente inmorales. Este sentimiento de amenaza es parte de la causa del aumento de la moralidad populista, descrita anteriormente. Sin embargo, para ser claros, la moralidad populista, que se hizo evidente a los pocos años de la reforma democrática, está aumentando no sólo en relación con el islam, sino que hay muchos otros factores. De hecho, se pueden encontrar muchos ejemplos de represión sexual en Indonesia y se pueden agrupar bajo el título de moralidad populista, como se explora a continuación. 


\section{Ejemplos de moralidad populista y disminución}

de los derechos sexuales

Si bien ha habido avances hacia los derechos sexuales en gran parte de Indonesia, en los primeros años de la reforma democrática se han observado grietas. Por ejemplo, en 2004 varias provincias habían tipificado como delito la prostitución, utilizando una definición amplia de prostitución que incluía la sexualidad entre personas del mismo sexo. Las provincias pudieron elaborar dichos estatutos aprovechando un sello distintivo de la reforma democrática: la descentralización, un proceso mediante el cual la toma de decisiones se transfiere del nivel nacional al nivel local. Durante el gobierno de Suharto hubo mucho descontento con los políticos de Yakarta, que tomaban decisiones sobre asuntos locales, frecuentemente, en contra de los intereses de los locales. La toma de decisiones a nivel local era algo que demandaba la gente en la nueva era democrática. Se creía que los líderes locales tomarían buenas decisiones para la población local. Sin embargo, en muchas ocasiones éste no fue el caso. Más bien, los líderes locales a menudo estaban aún interesados principalmente en su propio bienestar y aprobaban leyes para tal efecto. Se podría argumentar que la criminalización de la prostitución en varias provincias fue un ejemplo de líderes locales actuando en beneficio propio, a menudo con el fin de obtener apoyo para ser reelegidos.

Si bien las provincias locales estaban aprobando leyes que reducían los derechos sexuales en los primeros años del reformasi, no fue sino hasta 2008 que se aprobó una ley nacional, conocida como la Ley de Pornografía (Rinaldo 2013). Aunque ésta no era tan estricta como la que se propuso originalmente, aún existían severas restricciones a la expresión sexual. Parte del problema era que la pornografía tenía una definición tan amplia que casi cualquier cosa podía clasificarse como prostitución. Por ejemplo, el artículo 1 de la ley establece que la pornografía incluye "imágenes [...] conversaciones, movimientos del cuerpo [...] que contienen obscenidad (kecabulan) o explotación sexual que viola las normas morales (kesusilaan) de la sociedad" (Pausacker 2012; véanse también Davies 2018a; McRae 2018; Pausacker 2018). Como resultado de esta amplia definición, las minorías sexuales han sido perseguidas bajo ella (Renaldi 2017).

La Ley de Pornografía es quizá la más infame de las primeras leyes de restricción a la sexualidad, pero hubo muchos cambios menores que también la restringieron (Allen 2007). 
Muchas provincias criminalizaron la prostitución y la definieron de manera que incluyera la sexualidad entre personas del mismo sexo (Liang e IA 2016). Esta tendencia continuó durante muchos años (Liang e IA 2016) y culminó en las “crisis LGBT" en 2016 (Davies 2016), el mismo año en que el gobernador de Yakarta, Basuki Tjahaja Purnama, fue condenado por blasfemia (Fealy 2016). Las "crisis LGBT" atestiguaron la prohibición a los programas de radio y televisión para transmitir capítulos que presentaran lo LGBT como "normal", la disminución del financiamiento de la ONU para apoyar a las personas LGBT y las protestas anti-LGBT que la policía no pudo detener (Yulius 2017; Boellstorff 2016; Davies 2016).

Los derechos sexuales han seguido disminuyendo. En 2017, el Tribunal Constitucional rechazó una petición en una votación de 5/4 para ilegalizar las relaciones sexuales extramaritales. La Corte no necesariamente estuvo en desacuerdo con la petición, pero dijo que estaba fuera de su jurisdicción (Da Costa y Kapoor 2017). También ocurrieron otros eventos que mostraron que los derechos sexuales estaban disminuyendo en todo el archipiélago (Hajramurni 2017). En 2019 se sugirieron revisiones al Código Penal de Indonesia para ilegalizar todas las relaciones sexuales fuera del matrimonio. Este proyecto de ley fue recogido en los medios occidentales y fue coloquialmente llamado Legislación Anticópula de Bali ${ }^{3}$ (Mazzoni y Bevege 2019). El proyecto se pospuso en respuesta a protestas masivas (Gorbiano 2019; Reuters 2019), aunque la gente protestaba en gran medida por cambios distintos a los relacionados con la sexualidad. En 2020, el grupo conservador Alianza de Amor Familiar presentó otro proyecto de ley que nuevamente intentó tipificar como delito las relaciones sexuales fuera del matrimonio. Se llamó Proyecto de Ley de Resiliencia Familiar (Ketahanan Keluarga) e incluía todo tipo de propuestas draconianas, como exigir que una esposa ame a su esposo (Reuters 2020a). Muchos tienen la esperanza de que este proyecto no se convierta en ley (Yulisman 2020).

En los últimos años se han presentado pocos proyectos de ley que promuevan los derechos sexuales. Un ejemplo es el proyecto de ley contra la violencia sexual de 2020 (Indonesian Expat 2020). Éste criminalizaría toda la violencia sexual (Jumisih y Asmawaty 2020), pero ha habido una oposición sostenida al mismo. Por ejemplo, los detractores han dicho que, si se aprueba, el proyecto de ley destruirá la armonía marital y fomentará la

\footnotetext{
${ }^{3}$ Bali Bonking Bill.
} 
promiscuidad. Los opositores no han dado más detalles sobre cómo detener la violencia sexual destruirá los matrimonios, pero su retórica ha pospuesto con éxito la aprobación del proyecto de ley (Ramadhani, Fitri y Muryanto 2019). El caso de un hombre indonesio condenado por violación en serie en el Reino Unido ha echado más leña al fuego de los oponentes (Martin Anatias y Davies 2020; Neubauer 2020; Oetomo, Davies y Wijaya 2020; Reuters 2020b). Así, el movimiento de moralidad populista ha crecido durante las últimas dos décadas, $\mathrm{y}$, habiendo mostrado evidencia de ello, a continuación exploro algunos de los factores que sustentan la falta de derechos sexuales en Indonesia.

\section{Motores de la reducción de los derechos sexuales}

El causante más obvio de la disminución de los derechos sexuales en Indonesia es el poder y la influencia del islam conservador. Esta creciente influencia se evidencia en el hecho de que Ma'Ruf Amin, ex presidente del Consejo del Islam de Ulema, fue nombrado vicepresidente de Indonesia (2019-). El presidente Joko Widowo (2014-) no eligió originalmente a Ma'Ruf como su vicepresidente, pero fue una elección estratégica. El periodo reformasi había creado condiciones que hicieron de Ma'Ruf la persona perfecta para una candidatura electoral nacionalista-islámica equilibrada.

Bajo el gobierno autoritario del presidente Suharto (1965-1998) no se permitió al islam ejercer mucha influencia en la política (Rodríguez 2020). Así ocurrió también en los primeros años de reformasi, pero con la presidencia de Susilo Bambang Yudhoyono (20042014) la influencia islámica comenzó a crecer (Hoesterey 2013). En 2010 el Tribunal Constitucional de Indonesia aprobó la Ley de Blasfemia y su aprobación mostró que los partidos políticos islámicos y nacionalistas podían trabajar juntos con gran efecto (Bourchier 2019). Cuando llegaron las elecciones presidenciales de 2019, el islam conservador tuvo tal influencia que Ma’Ruf se convirtió en la elección obvia para vicepresidente.

Grupos islamistas influyentes también han trabajado para imponer restricciones a los derechos sexuales. Ha ejercido el poder el grupo militante Frente de Defensores Islámicos (Frente Pembela Islam, FPI), al igual que la Alianza de Amor Familiar (AILA). Esta última habla de construir familias fuertes y afirma que esto sólo se puede lograr criminalizando la sexualidad no marital; además ha tenido éxito en difundir este mensaje, en parte porque ha 
reclutado miembros influyentes, como profesores universitarios (Hermawan 2016). El poder y la influencia de AILA podrían indicar que, en general, los indonesios se están volviendo cada vez más intolerantes, pero hay investigaciones que sugieren que éste no es el caso (Mietzner 2020). Por el contrario, las investigaciones proponen que los musulmanes conservadores y con educación universitaria están llegando a posiciones de poder y están utilizando este poder para reducir los derechos sexuales. Entonces ¿cómo es el sistema educativo de Indonesia?

Indonesia implementó un nuevo plan de estudios en 2013 e incluyó algunos objetivos aceptables, como producir futuros ciudadanos "tolerantes, socialmente sensibles, democráticos y responsables" (Parker 2018). Pero una mirada más profunda a los planes de estudios muestra muchas contradicciones y revela que el sistema educativo de Indonesia no produce ciudadanos tolerantes. Por ejemplo, mientras que la materia "Educación ciudadana" parece "digna de elogio en su promoción de la tolerancia religiosa", la materia "Religión" tiene como objetivo aumentar "la piedad y la fe en lugar de la tolerancia interreligiosa" (Parker 2018). Dentro de los internados islámicos (pesantren) es probable que la situación produzca graduados menos tolerantes, especialmente porque los estudiantes no interactúan con los no musulmanes (Laksana 2020). También son motivo de preocupación las personas que apoyan la ilegalización de la sexualidad no matrimonial utilizando sus posiciones de poder, como profesores universitarios, para legitimar la homofobia (Martin Anatias y Davies 2020; Hermawan 2016). Si bien se carece de investigación, es posible que una de las razones por las que los indonesios altamente educados se sienten atraídos por puntos de vista intolerantes es que al adherirse a ellos son recompensados por bases de apoyo potenciales (por ejemplo, en el ámbito político, apelar a ciertos grupos al abrazar puntos de vista intolerantes da como resultado votos electorales en elecciones regionales y nacionales).

Otro factor educativo clave en la falta de derechos sexuales es la baja calidad de la información sobre salud sexual y reproductiva (Utomo et al. 2014). El nivel de desinformación sexual se mostró cuando un comisionado indonesio declaró públicamente en 2020 que un tipo de esperma podría embarazar a las mujeres en las piscinas (The Jakarta Post 2020). Esta información errónea no ayuda a los esfuerzos para aumentar los derechos sexuales (Sastramidjaja 2019). 
También hay causantes políticos que están reduciendo los derechos sexuales. En los primeros años de reformasi hubo signos de progreso. Por ejemplo, se otorgó mayor poder a la Comisión de Derechos Humanos y se inauguró la Comisión Nacional para la Violencia contra la Mujer. La violación conyugal se convirtió en delito (Andajani-Sutjahjo, Bennett y Davies 2018). Sin embargo, una de las ironías de la democracia es que, si bien los derechos de las minorías podrían estar teóricamente protegidos, se abre un espacio para la propagación del odio y el abuso. Después de dos décadas de democracia, Indonesia está siendo testigo de un estancamiento democrático impulsado por élites políticas y antirreformistas (Mietzner 2012), muchas de las cuales han resurgido de posiciones de poder anteriores (Bourchier 2019). El populismo y las innovaciones autoritarias también se están utilizando para reducir aún más los derechos sexuales (véanse Mietzner 2020; Mietzner 2012).

Como se señaló anteriormente, el populismo domina el panorama político contemporáneo de Indonesia (Mietzner 2020) y ha permitido a las personas en el poder señalar a quienes tienen relaciones sexuales fuera del matrimonio como inmorales (Kartika 2019). Los inmorales no son sólo sexualmente inmorales, además son posicionados como contrarios al Estado (Walton 2016b) y por haber formado un movimiento peligroso (gerakan) que libra una guerra de poder (Haripin 2016) contra las familias y la integridad nacional (Paramaditha 2016). La nostalgia por la llamada estabilidad del gobierno autoritario del presidente Suharto también se utiliza para aumentar el odio contra los "inmorales", a quienes se enmarca como la causa de los disturbios (Aspinall 2015). Una forma interesante en que el populismo ha reducido los derechos sexuales es a través de innovaciones autoritarias.

La innovación autoritaria es la idea de que los valores democráticos se han utilizado como una tapadera para ayudar a fines iliberales, al igual que el neoliberalismo se ha apoderado del feminismo (Davies 2019a). Los políticos, líderes religiosos y otros en el poder han reetiquetado las prácticas autoritarias como democráticas y las han presentado a las personas que continúan apoyando la democracia en Indonesia (Mietzner 2020). Podemos ver innovaciones autoritarias en todo tipo de casos. Por ejemplo, la discusión sobre las personas LGBT se ha movido, en parte, de una que señala que éstas deben ser perseguidas a una que señala que deben ser atendidas y rehabilitadas (Rodriguez 2020; Thajib 2020). Se han presentado proyectos de ley para prohibir las relaciones sexuales fuera del matrimonio como 
esfuerzos para salvar la integridad familiar. Los medios de comunicación de Indonesia han estado interesados en presentar historias en torno a esos ángulos y tienen una influencia increíble en Indonesia.

Casi la mitad de los indonesios, o alrededor de 132 millones de personas, son usuarios activos de las redes sociales (Kemp 2017). Muchos de estos usuarios son mujeres devotas (Nisa 2018). Las redes sociales, por supuesto, tienen el potencial de promover los derechos sexuales (Kirnandita 2020; Wijaya Mulya 2020; Winarnita et al. 2020), pero también pueden funcionar en contra de la igualdad sexual y la justicia (Lim 2017; véanse también Tapsell 2015). Por ejemplo, en 2016, un hashtag de tendencia en Twitter en Indonesia fue \#TolakLGBT (\#RechazoLGBT) (Liang e IA 2016). De hecho, a lo largo de 2016, las redes sociales de Indonesia se llenaron de discursos de odio LGBT (Listiorini 2020; Puteri y Wijaya 2020). Se prohibieron las plataformas de medios que habían proporcionado espacio para la solidaridad LGBT (Listiorini y Davies 2017), al igual que la palabra pelakor, un término que describe a una mujer que desafía el matrimonio de otra, a menudo encuadrada como una amante (Martin Anatias 2020), y los periódicos estudiantiles que compartían historias de amor lésbico (Pearson 2019). La vergüenza en las redes sociales deja un registro permanente (Lariat 2020; Davies 2018a).

Las redes sociales han ayudado a plantear una idea muy limitada de la persona moral. Como en todas partes, las nociones de moralidad están más fuertemente inscritas en el cuerpo de las mujeres, y en Indonesia el velo, una de las prendas de vestir más controvertidas del mundo, ha llegado a ser un significado de moralidad (Davies 2019b). Una encuesta de 2014 sugirió que 64\% de las mujeres indonesias que usan velo (Qibtiyah 2019; el porcentaje es probablemente más alto hoy), predicadores famosos (Kloos 2018), grupos de autoayuda (Jones 2010), el consumo conspicuo (Jones 2018), los signos de logro (Long 2013) y las economías espirituales neoliberales (Rudnyckyj 2009), todos ayudan a difundir el mensaje de que una persona moral no tiene relaciones sexuales fuera del matrimonio heterosexual (Heryanto 2011). Si no se está casado por vía heterosexual, y en muchos casos incluso si se está, no hay garantía de derechos sexuales.

Muchos factores se han unido en Indonesia para reducir los derechos sexuales. Arriba he examinado cuatro factores clave: religión, educación, política y redes sociales. Sólo 
examiné estos cuatro por razones de espacio y también porque son particularmente influyentes en la configuración de los derechos sexuales en Indonesia. Sin embargo, existen muchos otros factores que no hay espacio para discutir aquí, incluidos los legales (Outright Action International 2020; Crouch 2019; Butt 2018; Pausacker 2018), el patriarcado (McGregor, Dragojlovic y Loney 2020), los económicos (Allmark y Wahyudi 2019; Tadjoeddin y Chowdhury 2019; Toomistu 2019b; Piketty 2014; Wilson, Djani y Masduki 2009; Wilson 2006), el neoliberalismo (Trnka y Trundle 2014), la salud (Sarbini y Has 2019; Hegarty 2018; Hegarty 2017), los derechos humanos (McGregor y Setiawan 2019), la sociedad civil (Wieringa 2019), las restricciones a los activistas (Dibley y Ford 2019), el género, la globalización, el nacionalismo (Boellstorff 2016), las políticas de identidad (Purdey, Aspinall y As'ad 2016), el discurso (Thajib 2020), la cultura popular (Toomistu 2019b) y los medios de comunicación (Decker 2020; Murtagh 2013). Si bien el islam conservador es quizá el más influyente, todos estos factores se han combinado para impulsar la criminalización del sexo fuera del matrimonio y la reducción general de los derechos sexuales en todo el archipiélago. La gente ha encontrado formas de crear un espacio seguro (Rodriguez 2020; Thajib 2020; Hegarty 2017), pero se están reduciendo. Habiendo explorado ahora el contexto cultural en el que la moralidad populista está restringiendo los derechos sexuales, la segunda mitad del artículo explora el papel de la fuerza policial de Indonesia en la promoción de esta restricción a los derechos sexuales. Es mediante el examen del papel de la policía como podemos obtener una comprensión más completa de cómo el Estado, a través de su fuerza policial, limita los derechos sexuales.

\section{VIGILANCIA DE LA SEXUALIDAD}

\section{Fuerza policial de Indonesia}

En Indonesia la policía ha sido cooptada para restringir los derechos sexuales. Más que quedarse al margen y no actuar, existe evidencia de que la policía ha estado involucrada activamente en la persecución de miembros de la comunidad LGBT y otros grupos de minorías sexuales. Para comprender la falta de derechos sexuales en Indonesia, es imperativo comprender a la policía y su papel. 
Teniendo en cuenta que la fuerza policial de Indonesia es una de las más grandes del mundo, es sorprendente que se haya escrito poco sobre esta institución (Buttle, Davies y Meliala 2016; Meliala 2001a, 2001b, 2002). Uno de los escritores más prolíficos es Adrianus Meliala, ex comisionado de policía y profesor de la Universidad de Indonesia, quien señala que la policía de Indonesia se creó justo después de la Declaración de Independencia en 1945 (Meliala 2002) para ayudar al personal del ejército indonesio a luchar contra las fuerzas de ocupación holandesas y luego japonesas durante el periodo 1945-1948. Se tomaron medidas para asegurar que la policía fuera neutral y estuviera alejada de la influencia política, y para que disfrutara de autonomía operativa y administrativa en igualdad de condiciones con las fuerzas armadas. Después de que el régimen de Suharto asumió el poder, la policía se fusionó con las fuerzas armadas. Como tal, en 1968, Polri, el acrónimo por el que se conoce a la policía, era poco más que un brazo paramilitar de las fuerzas armadas que reprimía la disidencia contra el régimen autoritario del presidente Suharto. Como suele ser el caso, una fuerza policial organizada en la línea militar percibe a la población como el enemigo y utiliza formas brutales y, a veces mortales, de violencia extralegal (Waddington y Wright 2008). Es comprensible que tales acciones hagan que la gente desconfíe de la policía, lo que ha sucedido en Indonesia (IPAC 2013).

Desde la caída del régimen de Suharto en 1998, Polri ha experimentado una serie de reformas. Meliala (2002) indica que en 1999 la policía volvió a ser separada de los militares y esta separación significó que se restableció una mayor autonomía de la influencia política. Esta restauración fue ayudada por la descentralización de la gobernanza nacional a un nivel de administración local (Wilson, Djani y Masduki 2009). En muchos sentidos, la descentralización dio como resultado una mayor autonomía para las unidades de policía locales y aflojó el control nacional sobre la fuerza policial (Kristiansen y Trijono 2005). Pero Polri a menudo abusaba de su poder y la gente percibía cada vez más esta institución como poco confiable (Meliala 2002).

Como una forma de promover la legitimidad policial a los ojos de la población indonesia, Meliala (2001b) apoyó la idea de sensibilidad en la vigilancia. Así sugirió que había tres dimensiones de la sensibilidad policial que podrían introducirse y fomentarse en Indonesia: los agentes de policía individuales podrían ser sensibles en sus interacciones con 
la población en general; la cultura policial podría desarrollarse de una manera que promoviera sensibilidad a las necesidades públicas, y se podría garantizar que la dinámica organizativa de Polri promoviera un enfoque sensible de la actividad policial. Si estas cosas fueran posibles, la policía podría haberse convertido en una fuerza sensible en el área de la vigilancia sexual. Los derechos sexuales habrían tenido más posibilidades de ser garantizados si la policía hubiera aumentado su nivel de sensibilidad como lo ha promovido Meliala desde principios de 2000 .

Meliala también abogó por un enfoque de la policía orientado a la comunidad en Indonesia y, si esto hubiera sucedido junto con la necesidad de sensibilidad en la vigilancia, los derechos sexuales para los ciudadanos indonesios podrían haberse asegurado. De hecho, muchos estudios han destacado el potencial de la policía comunitaria en Indonesia y más allá; incluso apoyaron tentativamente su efectividad, especialmente cuando se trata de conflictos regionales (Prasetyo, Marzuki y Partnership for Governance Reform in Indonesia 2005).

En un intento de avanzar hacia la policía orientada a la comunidad, Polri hizo algunos esfuerzos. Por ejemplo, aprovechó el apoyo de la comunidad internacional para comenzar a alejarse de una comprensión paramilitar de la aplicación de la ley (Prasetyo, Marzuki y Partnership for Governance Reform in Indonesia 2005). Esta medida, si se adoptara más a fondo, mejoraría considerablemente la forma en que Polri se relaciona con la población, incluidas las minorías sexuales, especialmente en comparación con la forma en que el país fue vigilado por los militares durante el régimen del presidente Suharto. Tal compromiso mejorado vería probablemente un aumento en los derechos sexuales. Sin embargo, es discutible hasta qué punto Polri ha incorporado los valores de la policía comunitaria (Davies, Buttle y Meliala 2015).

La evidencia sugiere que una actuación policial eficaz requiere la cooperación de los ciudadanos (Alpert et al. 1998), pero los indonesios todavía desconfían en gran medida de la policía. Como tal, el movimiento de Polri sobre la vigilancia policial comunitaria ha fracasado mayormente porque dependía de crear la impresión de que la policía es parte de la comunidad (Hawdon y Ryan 2003). Hasta el día de hoy, pocas personas en Indonesia ven a Polri como parte de su comunidad (Davies, Buttle y Meliala 2015). Como tal, la vigilancia policial comunitaria efectiva, que tiene mucho que ver con la construcción de percepciones 
públicas positivas sobre la confiabilidad de la policía (Tyler y Huo 2002), ha experimentado reveses en Indonesia (Davies, Buttle y Meliala 2015).

Uno de los mayores contratiempos para mejorar la vigilancia policial en Indonesia es que la corrupción sigue siendo un problema endémico. Una teoría de restricciones culturales sugiere que la corrupción persiste en Indonesia debido, en parte, a la aprobación social tácita (Buttle, Davies y Meliala 2016). La corrupción es una barrera notable para la policía comunitaria (Pino y Johnson 2011) y la corrupción policial ha erosionado el apoyo de la comunidad a Polri en Indonesia (Davies y Buttle 2014; Meliala 2002).

A pesar de un mayor compromiso con ideas como la policía comunitaria, Polri sigue siendo principalmente una fuerza policial paramilitar y su relación con la población sigue siendo tensa. Muchos de los problemas a los que se ha enfrentado Indonesia con la violencia separatista, religiosa y étnica se han resuelto mediante técnicas informales de resolución de conflictos (Braithwaite et al. 2010), en lugar de recurrir a la policía o al sistema de justicia penal. Además, la proliferación de grupos de autodefensas, a menudo apoyados por intereses políticos (Wilson 2006), desafía la legitimidad de Polri y del sistema de justicia penal. Estos resultados decepcionantes en términos de vigilancia significan que la policía a menudo ha recibido una cobertura mediática desfavorable (Davies, Stone y Buttle 2016), tanto en los medios locales como nacionales (Davies, Stone y Buttle 2015). Dada la falta de responsabilidad de Polri, no es sorprendente que no haya garantía de los derechos sexuales en Indonesia. Pero el motivo de preocupación no es sólo la falta de interés de Polri por proteger a las minorías sexuales y a otras personas. La forma en que la policía recluta a las mujeres y los roles que luego se asignan a las mujeres policía también son razones de la falta de derechos sexuales en Indonesia (Davies, Meliala y Buttle 2016).

\section{Mujeres policía}

Para comprender la falta de derechos sexuales en Indonesia es importante tener en cuenta no sólo la institución de la policía, sino también el reclutamiento y el despliegue de mujeres policías. En resumen, las mujeres policía son reclutadas por su belleza, devoción y virginidad, y se despliegan para proteger la "moralidad" sexual de los ciudadanos de Indonesia. El movimiento de moralidad populista de Indonesia está impulsado hasta cierto punto por el 
particular estilo militar de la policía esbozado anteriormente, pero también por la forma de vigilancia policial, como se muestra a través de las mujeres policías.

Las mujeres han sido parte de Polri desde 1948, sólo un par de años después de que se desarrollara la fuerza policial. A pesar de este largo linaje, todavía hay muy pocas mujeres policía. Si bien Polri ha hablado de la esperanza de que al menos el 30\% de todos los agentes de policía sean mujeres, las mujeres policía actualmente representan apenas 5\% del número total de personal de Polri, que supera con creces el medio millón (Davies, Meliala y Buttle, 2016). Sin embargo, ha habido algunos incrementos en la cantidad de oportunidades de capacitación para mujeres (Human Rights Watch 2014). La mayoría de las mujeres policía trabajan sólo en algunas áreas, y éstas se encuentran principalmente en unidades que ayudan a mujeres y niños y en funciones administrativas.

Las mujeres policía indonesias eran en gran parte invisibles antes de 2013. Había poco material publicado sobre ellas en indonesio o inglés. Pero en 2013 comenzaron a ser más prominentes mientras estaban de servicio (por ejemplo, como policías de tránsito) y en las redes sociales. Por ejemplo, las mujeres policías han desempeñado un papel muy visible en la provisión de seguridad en eventos en los que se puede repartir dulces a las personas. También suelen tener un papel clave en la coordinación del tráfico y en la lectura de informes de tráfico para los medios de comunicación. Parte de este trabajo es potencialmente peligroso, pero Polri refuerza en la población la idea de que los policías siempre están ahí para ayudar a las mujeres policías si es necesario. Algunos de los indonesios más seguidos en Twitter son mujeres policía. Por ejemplo, la brigadier Eka Frestya tiene más de 30000 seguidores en Twitter. Para muchas mujeres jóvenes, convertirse en policía comenzó a aparecer como una opción profesional atractiva (Davies y Taylor-Alexander 2019).

Polri ha promovido la idea de que las mujeres policía bonitas, devotas y virginales pueden facilitar relaciones positivas entre la policía y el público. Para garantizar que las mujeres policía cumplan con estos ideales, existen estrictos criterios de reclutamiento (Davies 2015). Las solicitantes deben tener entre 17 y 22 años y ser solteras. Deben permanecer solteras durante dos años. Después de dos años pueden casarse $y$, con el permiso de su marido, seguir trabajando como oficiales de policía. Las solicitantes deben pasar pruebas psicológicas y tener fuertes creencias religiosas. Necesitan un certificado de 
preparatoria. No pueden usar anteojos. Tienen que aceptar ser reubicadas en cualquier lugar de Indonesia. Las solicitantes deben medir más de $165 \mathrm{~cm}$ de altura y su índice de masa corporal debe estar dentro del rango ideal. Deben ser atractivas (enak dilihat, literalmente "deliciosa a la vista"). La piel clara es con frecuencia una suposición implícita de ser bien parecido (Saraswati 2013).

Durante el proceso de reclutamiento se toman medidas corporales de las mujeres y, en ocasiones, los hombres miden el tamaño de busto de una mujer (Chanel Bombon 2014). A veces, las solicitantes deben caminar sobre un escenario frente a un comité de selección compuesto en su totalidad por policías hombres. Hay otro trabajo que se centra en la apariencia de las mujeres policía, enmarcándolo como el "imperativo de la belleza" (Davies y Hartono 2015). El argumento es que el imperativo de la belleza funciona no sólo para definir quién puede convertirse en mujer policía, sino también para definir los roles apropiados. Por ejemplo, a las mujeres policía reclutadas por motivos de belleza se les dice que son demasiado hermosas para emprender investigaciones penales. El tipo de persona atraída por la policía, en combinación con el proceso de reclutamiento, supone que las mujeres policía a menudo se ven impulsadas por el deseo de proteger la moralidad, especialmente la sexual, de los ciudadanos indonesios. A veces, las mujeres no tienen necesariamente clara su motivación inicial para unirse a la policía y algunas son abiertamente alentadas por su familia (Davies y Deckert 2020; Davies y Deckert 2019; Davies y Hartono 2015). Pero para muchas la motivación es hacer y mantener a Indonesia como una sociedad moral.

La importancia del velo y la virginidad para las mujeres policía muestra la importancia subyacente de la moralidad en Indonesia. Por ejemplo, en 2005, a las mujeres policía se les prohibió usar el velo musulmán (hijab) mientras estaban de servicio (Davies 2018b). En 2013, después de una reñida batalla, las mujeres policía musulmanas, basándose en parte en un discurso de derechos humanos, ganaron el derecho a usar velo en sus funciones (Hamida 2013). Sólo 12 meses después de esto, hubo un informe de Human Rights Watch en el que se señaló la práctica continua de las pruebas de virginidad para las mujeres como parte del reclutamiento policial (Human Rights Watch 2014). Estas llamadas pruebas de virginidad no son concluyentes ni científicas y, como tales, no "prueban" nada. Si bien 
algunas mujeres policías expresaron su disgusto por esta práctica, que ha estado en curso desde al menos 1965, muchas se sintieron orgullosas de ella y dijeron que era una forma de demostrar la moralidad sexual (Davies 2018b). Certificadas como sexualmente morales, las mujeres policía podrían posicionarse como capaces de vigilar la moralidad sexual (véase también Ridwan y Wu 2018). Juntos, estos eventos son indicativos de lo que subyace a la incapacidad de la policía para promover los derechos sexuales; la idea de que muy pocos ciudadanos son considerados realmente dignos de tal protección (Walton 2016a).

\section{Fracaso de la policía para proveer derechos sexuales}

Habiendo examinado el movimiento de moralidad populista y el apoyo generalizado para negar los derechos sexuales a quienes se consideran inmorales, y resumiendo la constitución de la fuerza policial cuyo cometido es promover la moralidad sexual, paso ahora a mostrar la mecánica de la negación de los derechos sexuales. En otras palabras, ¿cómo es que la policía no garantiza los derechos sexuales? Reviso a continuación varios casos adicionales para mostrar que la policía se niega a proteger los derechos sexuales o los socava activamente.

Si bien gran parte del enfoque respecto a la falta de derechos sexuales se ha centrado en la comunidad LGBT, hay ejemplos más amplios de fallas policiales respecto a los mismos derechos. Por ejemplo, en 2011, una docena de agentes de policía entraron sin previo aviso a una habitación de hotel en Lombok y exigieron a los ocupantes que presentaran un certificado de matrimonio. La pareja heterosexual respondió tartamudeando que no estaban casados. Luego, la policía los escoltó hasta un camión que los esperaba y los llevó de regreso al cuartel para interrogarlos. Tales redadas se han vuelto cada vez más comunes. La policía dice que están tratando de encontrar orang maksiat (personas inmorales) y pasangan mesum (parejas pecadoras, por ejemplo, no casadas), y la definición de estos términos ha crecido para incluir a cualquier persona que no esté casada heterosexualmente. Para agravar aún más el problema, los medios de comunicación suelen publicar fotografías que intensifican la vergüenza y disuaden futuros actos de este tipo.

Otro ejemplo de la falta de protección de los derechos sexuales por parte de la policía puede verse en la conferencia de Asia de la Asociación Internacional de Lesbianas y Gays (ILGA, por sus siglas en inglés), celebrada en Indonesia en 2010. Con la asistencia de más de 
150 activistas de 16 países asiáticos, fue un gran evento. Sin embargo, cuando llegaron los participantes, el grupo islamista Front Pembela Islam (FPI, Frente de Defensores Islámicos) presionó a la policía para que cancelara el permiso de la conferencia y así se hizo. Los participantes encontraron lugares alternativos para realizar eventos de la conferencia, pero los miembros del FPI los localizaron e incluso los siguieron a su hotel. La policía no ofreció protección (Poore, 2010a; Poore 2010b). Como señaló Poore, "la policía tiene la obligación de hacer cumplir el Estado de derecho: prevenir el acoso y las amenazas a la seguridad, arrestar a los manifestantes si es necesario por incitar o planificar la violencia, evacuar a la turba por invadir propiedad privada, exigir un permiso a los fundamentalistas si insisten en realizar una manifestación, y acordonarlos para que puedan ejercer su libertad de expresión sin obstaculizar la nuestra" (Poore, 2010a; Poore 2010b). Y lo que es más importante, "la policía debe proteger a las personas vulnerables de la violencia”. Sin embargo, la policía simplemente observó, proporcionando otro ejemplo de cómo hay una falta de derechos sexuales en Indonesia (véanse también BBC News 2011; Pausacker 2018; Suwarni y Dipa 2011).

\section{CONCLUSIÓN}

Este artículo ha expuesto que existe una restricción en los derechos sexuales en Indonesia, una restricción ocurrida de manera un tanto paradójica después de que Indonesia entró en la era de la democracia; fue la democracia la que en cierto sentido permitió a los votantes exigir restricciones de los derechos sexuales. Por lo tanto, los votantes han dado a la fuerza policial indonesia, en todos los niveles, el mandato de vigilar abiertamente y, en ocasiones, de manera punitiva los actos sexuales. Al mostrar cómo se dio este mandato a la policía, la primera mitad del artículo introdujo el concepto de moralidad populista y presentó ejemplos de las formas en que ésta se utiliza para justificar la persecución de expresiones de la sexualidad fuera de la heteronormatividad marital. La segunda parte del artículo examinó la institución de la policía y mostró cómo ésta emplea su posición de poder para reclutar mujeres policías devotas y virginales que luego son cooptadas para la vigilancia moral de la sociedad. Con tal mandato, la policía se siente justificada para ignorar los derechos sexuales y cometer injusticias sexuales. 
El impacto de la falta de derechos sexuales es devastador. Si bien los impactos principales se han sentido entre los miembros de la comunidad LGBT de Indonesia, las consecuencias se experimentan de manera más amplia (Agence France-Presse 2019). Por ejemplo, las madres casadas heterosexualmente, un grupo imaginado como ciudadanas morales, ha visto un aumento en el número de infecciones por VIH (Najmah, Davies y Andjani 2020). Parte de la razón de este aumento es que los esposos mantienen relaciones sexuales sin protección y transmiten el VIH y otras infecciones de transmisión sexual a sus esposas. Exigir o incluso practicar sexo seguro es difícil en Indonesia, donde los condones pueden usarse como prueba en los tribunales para condenar a alguien por actividad sexual fuera del matrimonio y, de hecho, incluso la compra de condones está restringida a las parejas casadas (Hajramurni 2020). En tal entorno, es también cada vez más difícil acceder a los servicios de salud reproductiva (Wilson 2020). Para un pleno florecimiento de la sociedad civil, los Estados (e instituciones como la policía, que mantiene las reglas) deben garantizar y apoyar los derechos sexuales.

Traducción: Yolanda González

Sharyn Graham Davies es directora del Monash Herb Feith Indonesian Engagement Center en la Universidad Monash, en Australia. Trabajó anteriormente en la Universidad Tecnológica de Auckland, en Nueva Zelanda. Ha publicado extensamente sobre seguridad, sexualidad y género en Indonesia.

sharyn.davies@,monash.edu

\section{REFERENCIAS}

Agence France-Presse (AFP). 2019. "Indonesia Ministries Ban Pregnant, LGBT Job Seekers: Report." The Straits Times, noviembre 22, 2019. https://www.straitstimes.com/ asia/se-asia/indonesia-ministries-ban-pregnant-lgbt-job-seekers-report

Allen, Pam. 2007. “Challenging diversity?: Indonesia’s anti-pornography bill.” Asian Studies Review 31 (2): 101-115. 
Allmark, Panizza e Irfan Wahyudi. 2019. "Travel, sexuality and female Indonesian domestic migrant workers in Hong Kong." Continuum 33 (5): 630-642.

Alpert, Geoffrey, Roger Dunham y Alpert Piquero, eds. 1998. "On the study of neighborhoods and the police." En Community policing: Contemporary readings, 309-326. Long Grove, Illinois: Waveland Press, Inc.

Andajani-Sutjahjo, Sari, Linda Rae Bennett y Sharyn Graham Davies. 2018. "Silent Strategies: The Legacy of Sexual Violence among Chinese Indonesians." Intersections - Gender and Sexuality in Asia and the Pacific 42 (agosto). http:// intersections.anu.edu.au/issue42/davies_etal.pdf

Aspinall, Edward. 2015. “Oligarchic populism: Prabowo Subianto’s challenge to Indonesian democracy." Indonesia 99: 1-28.

BBC News. 2011. "Indonesia Sex Tape Star is Jailed.” Asia Pacific, enero 31, 2011. https:// www.bbc.com/news/world-asia-pacific-12321215

Bennett, Linda R., Sari Andajani-Sutjahjo y Nurul I. Idrus. 2011. "Domestic violence in Nusa Tenggara Barat, Indonesia: Married women's definitions and experiences of violence in the home." The Asia Pacific Journal of Anthropology 12 (2): 146-163.

Boellstorff, Tom. 2005. The gay archipelago: Sexuality and nation in Indonesia. Princeton, Nueva Jersey: Princeton University Press.

Boellstorff, Tom. 2016. "Against State Straightism: Five Principles for Including LGBT.” EInternational Relations, marzo 21, 2016. https://www.e-ir.info/2016/03/21/againststate-straightism-five-principles-for-including-lgbt-indonesians

Bourchier, David M. 2019. "Two Decades of Ideological Contestation in Indonesia: From Democratic Cosmopolitanism to Religious Nationalism." Journal of Contemporary Asia 49 (5): 713-733.

Braithwaite, John, Michael Cookson, Valerie Braithwaite y Leah Dunn. 2010. Anomie and violence: Non-truth and reconciliation in Indonesian peacebuilding. Canberra: ANU Press. 
Butt, Simon. 2018. "Religious conservatism, Islamic criminal law and the judiciary in Indonesia: a tale of three courts." The Journal of Legal Pluralism and Unofficial Law 50 (3): 402-434.

Buttle, John W., Sharyn Graham Davies y Adrianus E. Meliala. 2016. “A cultural constraints theory of police corruption: Understanding the persistence of police corruption in contemporary Indonesia.” Australian \& New Zealand Journal of Criminology 49 (3): 437-454.

Chanel Bombon. 2014. "Woman police recruits undergo internal examinations as part of 'virginity tests' in Indonesia." YouTube: https://www.youtube.com/watch? $\underline{\mathrm{v}}=\mathrm{Q}$ lytmkI5ndY

Crouch, Melissa, ed. 2019. The Politics of Court Reform: Judicial Change and Legal Culture in Indonesia. Cambridge: Cambridge University Press.

Da Costa, Agustinus Beo y Kanupriya Kapoor. 2017. "Indonesia Court Rejects Petition to Bar Consensual Sex Outside Marriage.” Reuters, diciembre 14, 2017. https://www. reuters.com/article/us-indonesia-court-adultery/indonesia-court-rejects-petition-tobar-consensual-sex-outside-marriage-idUSKBN1E80BI

Davies, Sharyn Graham. 2015. "Beautiful virgins: The hard road to becoming an Indonesian policewoman." Asian Studies Association of Australia, abril 21, 2015. http://asaa. asn.au/beautiful-virgins-the-hard-road-to-becoming-an/

Davies, Sharyn Graham. 2016. "Indonesian Tolerance Under Strain as Anti-LGBT Furore Grows.” Asian Studies Association of Australia, marzo 21, 2016. http://asaa.asn. $\underline{\mathrm{au} / \text { indonesian-tolerance-under-strain-as-anti-lgbt-furore-grows }}$

Davies, Sharyn Graham. 2018a. "Gender and sexual plurality in Indonesia: Past and present." En Routledge Handbook of Contemporary Indonesia, editado por Robert Hefner, 322334. Londres: Routledge.

Davies, Sharyn Graham. 2018b. "Skins of moralidadity: Bio-borders, ephemeral citizenship and policing women in Indonesia." Asian Studies Review 42 (1): 69-88. 
Davies, Sharyn Graham. 2019a. "How a Populist Moralidadity Movement is Blocking a Law Against Sexual Violence in Indonesia." The Conversation, septiembre 19, 2019. https://theconversation.com/how-a-populist-moralidadity-movement-is-blocking-a$\underline{\text { law-against-sexual-violence-in-indonesia-analysis-123448 }}$

Davies, Sharyn Graham. 2019b. "I Chose Freely To Wear a Veil Just as Many Muslim Women Do.” Stuff New Zealand, abril 4, 2019. https://www.stuff.co.nz/national/christchurch-shooting/111757204/i-chose-freelyto-wear-a-veil-just-as-many-muslim-women-do

Davies, Sharyn Graham y J. W. Buttle. 2014. "Policing in Indonesia: exploring ways in which the legitimacy of the police may effect economic development and the prosperity of the Indonesian state.” Wellington: Ministry of Foreign Affairs and Trade.

Davies, Sharyn Graham, John Buttle y Adrianus Meliala. 2015. "If You Lose Your Goat: Public perceptions of police in Indonesia.” Journal of Social Science Research 6 (2): 1036-1046.

Davies, Sharyn Graham y Antje Deckert. 2019. "Pretty Strong Women: Ingenious Agency, Pink Gloves and Muay Thai.” Sociology of Sport Journal 36 (3): 213-223.

Davies, Sharyn Graham y Antje Deckert. 2020. "Muay Thai: Women, fighting, femininity." International Review for the Sociology of Sport 55 (3): 327-343.

Davies, Sharyn Graham, Adrianus Meliala y John Buttle. 2016. "Gangnam Style versus Eye of the Tiger: people, police and procedural justice in Indonesia." Policing and Society 26 (4): 453-474.

Davies, Sharyn Graham y Jazz Robson. 2016. "Juvenile (In) justice: Children in Conflict with the Law in Indonesia." Asia-Pacific Journal on Human Rights and the Law 17 (1): 119-147.

Davies, Sharyn Graham y Hanny Savitri Hartono. 2015. “The pretty imperative: Handcuffing policewomen in Indonesia." Intersections - Gender and Sexuality in Asia and the Pacific 37. http://intersections.anu.edu.au/issue37/davies hartono.htm 
Davies, Sharyn Graham, Louise M. Stone y John W. Buttle. 2015. “A Disinterested Press: Reporting police in a provincial Indonesian newspaper." Media Asia 42 (1-2): 47-60.

Davies, Sharyn Graham, Louise M. Stone y John Buttle. 2016. “Covering cops: Critical reporting of Indonesian police corruption.” Pacific Journalism Review 22 (2): 185.

Davies, Sharyn Graham y Sam Taylor-Alexander. 2019. "Temporal Orders and Y Chromosome Futures: Of Mice, Monkeys, and Men.” Catalyst: Feminism, Theory, Technoscience 5 (1): 1-18.

Davies, Sharyn Graham y Hendri Wijaya. 2019. "The unfulfilled promise of democracy: lesbian and gay activism in Indonesia." En Activists in Transition: Progressive Politics in Democratic Indonesia, editado por Thushara. Dibley y Michele Ford, 153170. Ithaca, Nueva York: Cornell University Press. https://doi.org/10.7591/ cornell/9781501742477.003.0009

Decker, Andrea. 2020. "Hidden for Their Protection: Gendered Power, Provocation, and Representation in Dangdut Competition Television.” Bijdragen tot de taal-, land-en volkenkunde/Journal of the Humanities and Social Sciences of Southeast Asia 176 (1): 37-69.

Dibley, Thushara y Michele Ford, eds. 2019. Activists in Transition: Progressive Politics in Democratic Indonesia. Ithaca, Nueva York: Cornell University Press.

Fealy, Greg. 2016. "Bigger than Ahok: Explaining the 2 December mass rally." Indonesia at Melbourne, The University of Melbourne, diciembre 7, 2016. https:// indonesiaatmelbourne.unimelb.edu.au/bigger-than-ahok-explaining-jakartas-2december-mass-rally/

Gorbiano, Marchio Irfan. 2019. "After Public Outcry Jokowi Demands Halt to Passing of Criminal Code Bill." The Jakarta Post, septiembre 20, 2019. https://www. thejakartapost.com/news/2019/09/20/after-public-outcry-jokowi-demands-halt-topassing-of-criminal-code-bill.html 
Hajramurni, Andi. 2017. "Police Ban Transgender Cultural Event in South Sulawesi." The Jakarta Pos, enero 20, 2017. https://www.thejakartapost.com/news/2017/01/20/ police-ban-transgender-cultural-event-in-south-sulawesi.html

Hajramurni, Andi. 2020. “Condoms are For Married People': Makassar Warns Shops, Drugstores Ahead of V-Day." The Jakarta Post, febrero 13, 2020. https://www.thejakartapost.com/news/2020/02/13/condoms-are-for-married-peoplemakassar-warns-shops-drugstores-ahead-of-v-day.html

Hamida. 2013. "Indonesian Police Allows Hijab." Al-Rasub. noviembre 25, 2013. https://www.alrasub.com/indonesian-police-allows-hijab/

Haripin, Muhamad. 2016. "Ryamizard's Proxy Wars." New Mandala, marzo 8, 2016. https://www.newmandala.org/ryamizards-proxy-wars/

Hawdon, James y John Ryan. 2003. "Police-resident interactions and satisfaction with police: An empirical test of community policing assertions." Criminal Justice Policy Review 14 (1): 55-74.

Hegarty, Benjamin. 2017. "Becoming Incomplete: The Transgender Body and National Modernity in New Order Indonesia (1967-1998)." Tesis, The Australian National University.

Hegarty, Benjamin. 2018. "Under the Lights, onto the Stage: Becoming Waria through National Glamour in New Order Indonesia." Transgender Studies Quarterly 5 (3): 355-377.

Hermawan, Ary. 2016. Commentary: Why AILA is a bigger threat to freedom than the FPI." The Jakarta Post, agosto 30, 2016. https://www.thejakartapost.com/news/2016/08/ $\underline{30 / \text { commentary-why-aila-is-a-bigger-threat-to-freedom-than-the-fpi.html }}$

Heryanto, Ariel. 2011."Upgraded piety and pleasure: The new middle class and Islam in Indonesian popular culture." En Islam and popular culture in Indonesia and Malaysia, editado por Andrew N. Weintraub, 76-98. Londres: Routledge. 
Hoesterey, James B. 2013. "Shaming the State: Subjectivity and Islamic Ethics in Indonesia's Pornography Debate." CURA-Luce Short Paper on Key Issues in Religion and World Affairs, Emory University.

Human Rights Watch. 2014. “Indonesia: 'Virginity Tests' for female police.” Noviembre 17, 2014. https://www.hrw.org/news/2014/11/17/indonesia-virginity-tests-female-police

Indonesia Expat. 2020. “Anti-Sexual Violence Law Will Be Passed.” PT Koleksi Klasik Indonesia, enero 19, 2020. https://indonesiaexpat.biz/featured/anti-sexual-violencelaw-will-be-passed/

Institute for Policy Analysis of Conflict (IPAC). 2013. "A Note on Recent Police Shootings Around Jakarta." Institute for Policy Analysis of Conflict, enero 20, 2013. http://www.understandingconflict.org/en/conflict/read/19/A-NOTE-ON-RECENTPOLICE-SHOOTINGS-AROUND-JAKARTA

Jones, Carla. 2010. "Better women: The cultural politics of gendered expertise in Indonesia." American Anthropologist 112 (2): 270-282.

Jones, Carla. 2018. "Consumption and the new middle classes." En Routledge Handbook of Contemporary Indonesia, editado por Robert Hefner, 187-196. Londres: Routledge.

Jumisih y Andi Cipta Asmawaty. 2020. "Sexual Violence and Women Workers.” Inside Indonesia, enero 29, 2020. https://www.insideindonesia.org/sexual-violence-andwomen-workers

Kartika, Dyah Ayu. 2019. “An Anti-Feminist Wave in Indonesia’s Election?” New Mandala, abril 14, 2019. https://www.newmandala.org/an-anti-feminist-wave-in-indonesiaselection/

Kemp, Simon. 2017. "Digital in Southeast Asia in 2017." We Are Social, febrero 16, 2017. https://wearesocial.com/special-reports/digital-southeast-asia-2017

Kirnandita, Patresia. 2020. “\#EroticArt: Agency, Advocacy and Women's Sexuality.” Inside Indonesia, enero 26, 2020. https://www.insideindonesia.org/eroticart-agencyadvocacy-and-women-s-sexuality-2 
Kloos, David. 2018. “Hearing Allah’s Call: Preaching and Performance in Indonesian Islam, by Julian Millie." Bijdragen tot de taal-, land-en volkenkunde/Journal of the Humanities and Social Sciences of Southeast Asia 174 (4): 517-520.

Kristiansen, Stein y Lambang Trijono. 2005. "Authority and law enforcement: local government reforms and security systems in Indonesia." Contemporary Southeast Asia: A Journal of International and Strategic Affairs 27 (2): 236-254.

Laksana, Ben K. C. 2020. "How the Daily Lives of Indonesian Youth Can Tell Us Why They Become More Conservative." The Conversation, marzo 2, 2020. https://theconversation.com/how-the-daily-lives-of-indonesian-youth-can-tell-uswhy-they-become-more-conservative-132019

Lariat, Joni. 2020. "Witnessing Shame and Punishment." Inside Indonesia, enero 26, 2020. https://www.insideindonesia.org/witnessing-shame-and-punishment

Liang, Jamison, e IA. 2016. "Moralidadity and LGBT Rights in Indonesia.” New Mandala, marzo 16, 2016. https://www.newmandala.org/moralidadity-and-lgbt-rights-inindonesia

Lim, Merlyna. 2017. "Freedom to hate: social media, algorithmic enclaves, and the rise of tribal nationalism in Indonesia." Critical Asian Studies 49 (3): 411-427.

Listiorini, Dina. 2020. “Online Hate Speech.” Inside Indonesia, enero 26, 2020. https://www.insideindonesia.org/online-hate-speech

Listiorini, Dina y Sharyn Graham Davies. 2017. “Online Dating Apps Blocked.” Inside Indonesia, abril 18, 2017. https://www.insideindonesia.org/online-dating-appsblocked

Long, Nicholas J. 2013. Being Malay in Indonesia: Histories, Hopes and Citizenship in the Riau Archipelago. Singapur: NUS Press.

Martin Anatias, Nelly. 2020. "Pelakor and Instagram 2.” Inside Indonesia, enero 20, 2020. https://www.insideindonesia.org/pelakor-and-instagram-2 
Martin Anatias, Nelly y Sharyn Graham Davies. 2020. “Opinions Should Never Be Dressed Up as Fact: Indonesian Media and Disinformation.” Jakarta Globe, febrero 25, 2020. https://jakartaglobe.id/opinion/opinions-should-never-be-dressed-up-as-scientificfact-indonesian-media-and-disinformation

Mazzoni, Alana y Alison Bevege. 2019. "Bali Bonking Ban Panic: Australians Cancel Their Holidays to the Resort Island Because They Fear Being Arrested Over New Laws Outlawing Sex Before Marriage.” MSN News, septiembre 20, 2019. https://www. msn.com/en-au/news/australia/bali-bonking-ban-panic-australians-cancel-theirholidays-to-the-resort-island-because-they-fear-being-arrested-over-new-lawsoutlawing-sex-before-marriage/ar-AAHAW7E

McGregor, Katharine, Ana Dragojlovic y Hannah Loney, eds. 2020. Gender, Violence and Power in Indonesia: Across Time and Space. Londres: Routledge.

McGregor, Katharine y Ken Setiawan. 2019. 'Shifting from International to 'Indonesian' Justice Measures: Two Decades of Addressing Past Human Rights Violations." Journal of Contemporary Asia 49 (5): 837-861.

McRae, Dave. 2018. "Talking Indonesia: Pornography." Indonesia at Melbourne, The University of Melbourne, enero 18, 2018. https://indonesiaatmelbourne. unimelb.edu.au/talking-indonesia-pornography

McWilliams, Susan. 2016. "This Political Theorist Predicted the Rise of Trumpism. His name was Hunter S. Thompson.” The Nation, diciembre 15, 2016. https://www.thenation.com/article/archive/this-political-theorist-predicted-the-riseof-trumpism-his-name-was-hunter-s-thompson/

Meliala, Adrianus. 2001a. "Police as military: Indonesia's experience." Policing: An International Journal of Police Strategies \& Management 4 (3): 420-432.

Meliala, Adrianus. 2001b. "The notion of sensitivity in policing." International Journal of the Sociology of Law 29 (2): 99-111.

Meliala, Adrianus. 2002. "Local colours for Indonesian national police.” Policing and society 12 (2): 153-161. 
Mietzner, Marcus. 2012. “Indonesia's democratic stagnation: anti-reformist elites and resilient civil society." Democratization 19 (2): 209-229.

Mietzner, Marcus. 2020. "Rival populisms and the democratic crisis in Indonesia: chauvinists, Islamists and technocrats.” Australian Journal of International Affairs 74 (2): 1-19.

Murtagh, Ben. 2013. Genders and sexualities in Indonesian cinema: Constructing gay, lesbi and waria identities on screen. Londres: Routledge.

Najmah, Sharyn Graham Davies y Sari Andjani. 2020. "Wives and Mothers and HIV." Inside Indonesia, enero 26, 2020. https://www.insideindonesia.org/wives-and-mothers-and$\underline{\text { hiv }}$

Neubauer, Ian Lloyd. 2020. “'It's Dangerous”: Sinaga Case Fuels LGBT Blacklash in Indonesia." Al Jazeera, enero 27, 2020. https:/www.aljazeera.com/news/ 2020/01/dangerous-sinaga-case-fuels-lgbt-backlash-indonesia$\underline{200125232137541 . h t m l}$

Nisa, Eva F. 2018. "Social media and the birth of an Islamic social movement: ODOJ (One Day One Juz) in contemporary Indonesia." Indonesia and the Malay World 46 (134): 24-43.

Oetomo, Dede, Hendri Yulius y Sharyn Davies. 2020. "Rejecting Homophobic Pseudoscience." The Jakarta Post, enero 30, 2020. https://www.thejakartapost.com/academia/2020/01/30/rejecting-homophobicpseudoscience.html

Outright Action International. 2020. "Creeping Criminalisation list of articles." https:// outrightinternational.org/search/node/Creeping $\% 20 \mathrm{criminalisation}$

Paddock, Richard C. y Muktita Suhartono. 2019. "A Test for Foreign Teachers in Indonesia: Are You Gay?" The New York Times, diciembre 23, 2019. https://www.nytimes. $\underline{\text { com/2019/12/23/world/asia/indonesia-gay-teachers.html }}$ 
Paramaditha, Intan. 2016. "The LBGT Debate and the Fear of 'Gerakan'." The Jakarta Post, febrero 27, 2016. https:/www.thejakartapost.com/news/2016/02/27/the-lgbt-debateand-fear-gerakan.html

Parker, Lyn. 2018. "The new curriculum in Indonesia.” Routledge International Handbook of Multicultural Education Research in Asia Pacific.

Pausacker, Helen. 2012. 'Playboy, the Islamic Defenders' Front and the Law: Enforcing Islamic Norms in Post-Soeharto Indonesia?” Australian Journal of Asian Law 13 (1).

Pausacker, Helen. 2018. Entrevista con Dave McRae. "Pornography." Talking Indonesia, podcast audio, enero 18, 2018. https://soundcloud.com/talking-indonesia/dr-helenpausacker-pornography

Pearson, Elaine. 2019. "Indonesian University Censors Lesbian Love Story.” Human Rights Watch, marzo 26, 2019. https://www.hrw.org/news/2019/03/26/indonesianuniversity-censors-lesbian-love-story

Piketty, Thomas. 2014. Capital in the 21st Century. Cambridge: Harvard University Press.

Pino, Nathan W. y Lee Michael Johnson. 2011. "Police deviance and community relations in Trinidad and Tobago." Policing: An International Journal of Police Strategies \& Management 34 (3): 454-478.

Poore, Grace. 2010a. "LGBT Activism Under Attack in Surabaya, Indonesia. Part One." International Gay and Lesbian Human Rights Commission (IGLHRC).

Poore, Grace. 2010b. "LGBT Activism Under Attack in Surabaya, Indonesia. Part Two." International Gay and Lesbian Human Rights Commission (IGLHRC).

Prasetyo, Eko, Supraman Marzuki y Partnership for Governance Reform in Indonesia. 2005. Evaluation report on community policing related project. Kepolisian Negara Republik Indonesia.

Prasetyo, E., B. Muqoddas, S. Marzuki, E. Riyadi, L. Arham, Imran y Soekamid. 2005. “The role of the police in socio-political conflicts in Indonesia." Pusham UII, The Asia Foundation, Yogyakarta. 
Purdey, Jemma, Edward Aspinall y Muhammad Uhaib As’ad. 2016. “Understanding family politics: Successes and failures of political dynasties in regional Indonesia." South East Asia Research 24 (3): 420-435.

Puteri, Made Diah Pitaloka Negara y Firdhan Aria Wijaya. 2020. “Mobilising Fear.” Inside Indonesia, enero 20, 2020. https://www.insideindonesia.org/mobilising-fear-2

Putri, K. Amanda, Shaila Tieken, Sharyn Davies Graham y Santi Kusumaningrum. 2019. “The Juvenile Courts and Children’s Rights: Good intentions, Flawed Execution.” En The Politics of Court Reform: Judicial Change and Legal Culture in Indonesia, editado por Melissa Crouch, 267-286. Cambridge: Cambridge University Press.

Qibtiyah, Alimatul. 2019. "Hijab in Indonesia - the History and Controversies." The Conversation, febrero 22, 2019. https://theconversation.com/hijab-in-indonesia-thehistory-and-controversies-102911

Ramadhani, Nurul Fitri y Bambang Muryanto. 2019. "House Pressured into Pledge to Rush Deliberation of Anti-Rape Bill.” The Jakarta Post, marzo 10, 2019. https:/www. thejakartapost.com/news/2019/03/10/house-pressured-into-pledge-to-rushdeliberation-of-antirape-bill.html

Renaldi, Adi. 2017. "Queer Indonesians Are Being Arrested Under a Vague Anti-Porn Law." VICE, junio 1, 2017. https:/www.vice.com/en_nz/article/j5ey9p/queer-indonesiansare-being-arrested-under-a-vague-anti-porn-law

Reuters. 2019. “Thousands Rally in Indonesia Amid Controversial Criminal Code Changes.” The Straits Times, septiembre 24, 2019. https://www.straitstimes.com/asia/seasia/thousands-rally-in-indonesia-amid-controversial-criminal-code-changes

Reuters. 2020a. "Indonesia Bill on Family Targets Surrogacy, 'Sexual Deviations'." The Straits Times, febrero 19, 2020. https://www.straitstimes.com/asia/east-asia/ indonesia-bill-on-family-targets-surrogacy-sexual-deviations

Reuters. 2020b. "Indonesia Rights Body Condemns Plans for LGBT Raids Ordered by Mayor After UK Rape Case.” The Straits Times, enero 14, 2020. https://www.straitstimes. 
com/asia/se-asia/indonesia-rights-body-condemns-lgbt-raids-ordered-by-mayorafter-uk-rape-case

Ridwan, Rinaldi y Joyce Wu. 2018. “'Being young and LGBT, what could be worse?' Analysis of youth LGBT activism in Indonesia: challenges and ways forward." Gender \& Development 26 (1): 121-138.

Rinaldo, Rachel. 2013. Mobilizing piety: Islam and feminism in Indonesia. Oxford: Oxford University Press.

Rodriguez, Diego Garcia. 2020. "Allies in Java." Inside Indonesia, enero 26, 2020. https://www.insideindonesia.org/allies-in-java-2

Rudnyckyj, Daromir. 2009. "Spiritual economies: Islam and neoliberalism in contemporary Indonesia." Cultural anthropology 24 (1): 104-141.

Rudnyckyj, Daromir. 2011. Spiritual economies: Islam, globalization, and the afterlife of development. Cornell University Press.

Saraswati, L. Ayu. 2013. Seeing beauty, sensing race in transnational Indonesia. University of Hawaii Press.

Sarbini, Firmansyah y Muh. Wildan Teddy Bintang P. Has. 2019. "Depok and its effort to criminalise the LGBT community." Australian Journal of Human Rights 25 (3): 520526.

Sastramidjaja, Yatun. 2019. "Chapter One. Student Movements and Indonesia's Democratic Transition." En Activists in Transition, editado por Thushara Dibley y Michele Ford, 23-40. Ithaca, Nueva York: Cornell University Press.

Suwarni, Yuli Tri y Arya Dipa. 2011. "Ariel Gets 3.5 Years; Unrest Outside Court.” The Jakarta Post, febrero 1, 2011. https://www.thejakartapost.com/news/2011/02/01/ ariel-gets-35-years-unrest-outside-court.html

Tadjoeddin, Mohammad Zulfan y Anis Chowdhury. 2019. Employment and Reindustrialisation in Post Soeharto Indonesia. Londres y Nueva York: Palgrave Macmillan. 
Tapsell, Ross. 2015. "Indonesia's media oligarchy and the 'Jokowi phenomenon'.” Indonesia 99: 29-50.

Thajib, Ferdiansyah. 2020. "Queering Piety." Inside Indonesia, enero 26, 2020. https://www.insideindonesia.org/queering-piety

The Jakarta Post. 2020. "Child Protection Commissioner Apologies for Saying 'Strong Sperm' Could Impregnant Women in Swimming Pools." TheJakartaPost.com, febrero 23, 2020. https:/www.thejakartapost.com/news/2020/02/23/childprotection-commissioner-apologizes-for-saying-strong-sperm-could-impregnatewomen-in-swimming-pools.html

Toomistu, Terje. 2019a. "Embodied Notions of Belonging: Practices of Beauty among Waria in West Papua, Indonesia.” Asian Studies Review 43 (4): 581-599.

Toomistu, Terje. 2019b. "Playground love: sex work, pleasure, and self-affirmation in the urban nightlife of Indonesian waria.” Culture, Health \& Sexuality 21 (2): 205-218.

Towner, Nicholas y Sharyn Davies. 2019. "Surfing tourism and community in Indonesia." Journal of Tourism and Cultural Change 17 (5): 642-661.

Trnka, Susanna y Catherine Trundle. 2014. "Competing responsibilities: Moving beyond neoliberal responsibilisation.” Anthropological Forum 24 (2): 136-153.

Tyler, Tom R. y Yuen Huo. 2002. Trust in the law: Encouraging public cooperation with the police and courts. Nueva York: Russell Sage Foundation.

Utomo, Iwu Dwisetyani, Peter McDonald, Anna Reimondos, Ariane Utomo y Terence H. Hull. 2014. "Do primary students understand how pregnancy can occur? A comparison of students in Jakarta, West Java, West Nusa Tenggara and South Sulawesi, Indonesia.” Sex Education 14 (1): 95-109.

Waddington, Peter A. J. y Martin Wright. 2008. "Police use of force, firearms and riot control." En Handbook of Policing, editado por Tim Newburn, 465-496. Londres: Routledge. 
Walton, Kate. 2016a. “'Lesbianism' and detention in Aceh.” New Mandala, octubre 9, 2016. https://www.newmandala.org/lesbianism-and-detention-in-aceh/

Walton, Kate. 2016b. "Unity in (Sexual) Diversity?" New Mandala, febrero 4, 2016. https://www.newmandala.org/unity-in-sexual-diversity/

Wieringa, Saskia E. 2019. "Criminalisation of Homosexuality in Indonesia: The Role of the Constitution and Civil Society." Australian Journal of Asian Law 20 (1): 227-245.

Wijaya Mulya, Teguh. 2020. “Queer Pop Culture.” Inside Indonesia, enero 20, 2020. https:// www.insideindonesia.org/queer-pop-culture-2

Wilson, Ian. 2006. "Continuity and change: The changing contours of organized violence in post-New Order Indonesia.” Critical Asian Studies 38 (2): 265-297.

Wilson, Ian. 2020. “Covid-19, Inequality and Jakarta's Urban Poor: Resilient, But at Great Risk." Indonesia at Melbourne, The University of Melbourne, abril 11, 2020. https://indonesiaatmelbourne.unimelb.edu.au/covid-19-inequality-and-jakartasurban-poor-resilient-but-at-great-risk/

Wilson, Ian, Luky Djani y Teten Masduki. 2009. “Governing favours': An investigation of accountability mechanisms in local government budget allocation in Indonesia." Policy Brief 8, Australia Indonesia Governance Research Partnership.

Winarnita, Monika, Nasya Bahfen, Gavin Height, Joanne Bryne y Adriana Rahajeng Mintarsih. 2020. “\#MeToo Indonesia.” Inside Indonesia, enero 26, 2020. https:// www.insideindonesia.org/metoo-indonesia

Yulisman, Linda. 2020. “Indonesia's Family Resilience Bill May See Uphill Battle for Deliberation as Opposition Mounts.” The Straits Times, marzo 13, 2020. https:// www.straitstimes.com/asia/se-asia/indonesias-family-resilience-bill-may-see-uphillbattle-for-deliberation-as-opposition

Yulius, Hendri. 2017. "The Dark Side of LGBT Awareness in Indonesia.” The Jakarta Post, octubre 9，2017. https://www.thejakartapost.com/academia/2017/10/09/the-darkside-of-lgbt-awareness-in-indonesia.html 